\title{
Desigualdad de los ingresos en el Brasil. ¿Qué ha cambiado en los últimos años?
}

\author{
Helder Ferreira de Mendonça y Diogo Martins Esteves
}

RESUMEN

En este estudio se presentan datos empíricos que permiten evaluar la repercusión de diversas variables socioeconómicas y políticas en distintas formas de medir la desigualdad de los ingresos en las 27 unidades de la República Federativa del Brasil entre 1999 y 2008. La experiencia brasileña resulta útil para entender las políticas relativas a la desigualdad de los ingresos en los países en desarrollo. Los resultados indican que el progreso observado durante el período analizado se debe a la combinación de una mayor apertura comercial, el desarrollo tecnológico y financiero, la reducción de la tasa de desempleo, la puesta en práctica de políticas sociales con efecto directo en las familias más pobres y la implementación de mecanismos contra la corrupción.

PALABRAS CLAVE

CLASIFICACIÓN JEL

AUTORES
Condiciones económicas, ingresos, distribución del ingreso, aspectos económicos, política social, medición, modelos econométricos, Brasil

D31, I32, R10

Helder Ferreira de Mendonça es profesor de economía de la Universidad Federal Fluminense e investigador del Consejo Nacional de Desarrollo Científico y Tecnológico. helderfm@hotmail.com

Diogo Martins Esteves es doctorando de la Universidad Federal Fluminense en el Departamento de Economía. diogomartinse@yahoo.com.br 


\section{I}

\section{Introducción}

El análisis de las principales causas de la desigualdad de los ingresos resulta esencial para descubrir formas de mitigarla. En la bibliografía sobre la desigualdad de los ingresos suele plantearse la cuestión de la repercusión de un aumento de la apertura comercial en la distribución de los ingresos. En las economías en desarrollo, donde existen altos niveles de desigualdad y predomina la mano de obra no calificada, un incremento de la apertura comercial puede provocar el crecimiento de las exportaciones, que - a su vez - hace que se reduzca la desigualdad de ingresos (Easterly, 2005). Como indican Nissanke y Thorbecke (2006), el avance de la apertura comercial suele verse acompañado del incremento de la inversión extranjera directa (IED) y la difusión de nuevas tecnologías y conocimientos especializados. Esto debería dar lugar a un aumento de la productividad y del producto que permita mejorar los salarios y el empleo ${ }^{1}$.

El proceso de mundialización también afecta a la distribución de los ingresos. Según Adams (2008), el uso de patentes como mecanismo de defensa de los derechos de propiedad intelectual y para estimular la innovación en las economías en desarrollo debería suponer una reducción de la desigualdad. Acemoglu y Newman (2002) observan el mismo resultado. Según esta opinión, en las economías en desarrollo es abundante la mano de obra no calificada y, por lo tanto, el progreso tecnológico hace que se incremente la productividad. En consecuencia, la difusión del conocimiento podría permitir un acrecentamiento de los ingresos de los trabajadores no calificados (Fang, Huang y Wang, 2008) y fomentar su mejor distribución.

El desempleo constituye, sin duda, la causa principal de la pobreza. Blinder y Esaki (1978) describieron por primera vez la relación entre el desempleo y la desigualdad de los ingresos. La idea fundamental es que el desempleo tiende a afectar más a las personas no calificadas y con salarios bajos que a otros grupos. De este modo, el desempleo suele concentrarse en el extremo inferior de la distribución de ingresos (Martínez, Ayala y Ruiz-Huerta, 2001).

El éxito de las políticas sociales resulta polémico para la bibliografía relacionada con ese tema. Hay datos

\footnotetext{
${ }^{1}$ Véase en Meschi y Vivarelli (2009) un análisis empírico contrario a la tesis de que la apertura comercial reduce la desigualdad de los ingresos.
}

empíricos que denotan que el aumento del salario mínimo es una poderosa herramienta para combatir la desigualdad de los ingresos (Lemos, 2009). Además, como señalan Engel, Galetovic y Raddatz (1999) y Goñi, López y Servén (2011), las transferencias gubernamentales constituyen un mecanismo eficiente para reducir la desigualdad. En cambio, Feldstein (1974) sostiene que el gasto social (servicios sociales, seguridad social y otros conceptos similares) acrecienta la desigualdad de los ingresos, ya que las familias con mayores rentas reciben un porcentaje desproporcionadamente alto de las prestaciones (Forteza y Rossi, 2009).

También resulta relevante el efecto de la competencia política. El estudio de la relación entre variables sociales y políticas se remonta a Adelman y Morris (1965). Como señalan Rupasingha y Goetz (2007), una mayor competencia política da lugar a un menor nivel de pobreza. La corrupción también se vincula a la desigualdad de los ingresos ya que su aumento provoca un incremento de dicha desigualdad (Gupta, Davoodi y Alonso-Terme, 2002; Dincer y Gunalp, 2008; Apergis, Dincer y Payne, 2010).

En este estudio se analizan los temas mencionados en el contexto del caso brasileño. El Brasil es uno de los principales países emergentes, con la séptima mayor economía del mundo, y recientemente ha tenido éxito en reducir la desigualdad de los ingresos. Esta última etapa ha estado marcada por una combinación de consolidación de la democracia, un entorno macroeconómico estable y la puesta en práctica de varias iniciativas contra la pobreza por parte del gobierno (por ejemplo, la implementación del programa Bolsa Família de transferencias condicionales y aumentos reales sucesivos del salario mínimo).

Al igual que los Estados Unidos de América, el Brasil se caracteriza por tener grandes diferencias geográficas. En consecuencia, el primer paso de este estudio consiste en observar el comportamiento de los factores principales que pueden explicar la desigualdad de los ingresos para cada región, identificando dónde cada factor tiene más influencia. El segundo paso es la presentación de datos empíricos, utilizando un marco dinámico de datos de panel que cubre las 27 unidades de la República Federativa del Brasil entre 1999 y 2008. En resumen, el objetivo principal de esta investigación es, mediante la presentación de datos empíricos, evaluar 
la repercusión de las variables socioeconómicas y políticas en diversas formas de medir la desigualdad de los ingresos.

El resto del estudio se organiza como se indica a continuación. En la sección II se describen los datos utilizados en esta investigación y se proporciona un análisis regional del caso del Brasil. En la sección III se presentan pruebas empíricas — basadas en el análisis de datos de panel- de la repercusión de las variables socioeconómicas y políticas en diferentes formas de medir la desigualdad de los ingresos en las 27 unidades de la República Federativa del Brasil entre 1999 y 2008. En la sección IV y última se entregan las conclusiones del estudio.

\section{II}

\section{Desigualdad de los ingresos: análisis regionales}

En 1999, el Brasil puso en práctica un régimen cambiario flexible, el establecimiento de objetivos de inflación y un superávit fiscal primario. La estabilidad macroeconómica resultante ha permitido que mejore la distribución de los ingresos. En este estudio se consideran diversas variables socioeconómicas (Roine, Vlachos y Waldenström, 2009; Easterly, 2005; Acemoglu, 2002) y políticas (Gupta, Davoodi y Alonso-Terme, 2002; Alt y Lassen, 2010), siguiendo la bibliografía sobre desigualdad de los ingresos. La enorme extensión del Brasil supone que las diferencias regionales también deben tenerse en cuenta. Por lo tanto, en esta sección se muestra el comportamiento regional de las variables utilizadas en el modelo empírico para el período comprendido entre 1999 y $2008^{2}$.

Durante décadas, la desigualdad de los ingresos en el Brasil ha sido muy grande en comparación con otros países (Gasparini, 2003). Sin embargo, esta comenzó a disminuir a finales de la década de 1990. Para delinear esta tendencia y comprobar su solidez, en el estudio se tienen en cuenta tres indicadores:

- $\quad$ El índice de desigualdad de Gini, que mide la desigualdad de una distribución en una escala del cero (igualdad plena) al uno (máxima desigualdad). Este coeficiente se calcula utilizando información disponible en la Encuesta Mensual de Empleo realizada por el Instituto Brasileño de Geografía y Estadística (IBGE).

- $\quad$ El índice de Theil, que mide la desigualdad económica en una escala del cero (igualdad plena) al uno (máxima desigualdad). Este índice se calcula mediante la información de la Encuesta Mensual de Empleo del IBGE.

\footnotetext{
${ }^{2}$ El período analizado finaliza en 2008 , debido a la falta de datos posteriores.
}

- La proporción entre los ingresos del $10 \%$ más rico de la población y los ingresos del $40 \%$ más pobre (el índice 10/40). Este índice se calcula sobre la base de la información de la Encuesta nacional de hogares del IBGE y se puede interpretar como una expresión de los ingresos de la población más rica como múltiplo de los ingresos de la población más pobre.

El comportamiento de estos indicadores a lo largo del tiempo indica que los niveles de vida mejoraron en todas las regiones. Todos los indicadores se redujeron al final del período considerado. En el gráfico 1 se aprecia que la región nordeste sufrió los peores niveles de desigualdad según todos los índices y a lo largo de la mayor parte del período (es decir, la línea de esta región es la más lejana del centro en todos los paneles del gráfico 1), pero registró un avance al final de la etapa, cuando la región centro-oeste pasó al último lugar. La región centro-oeste obtuvo los peores resultados de reducción de la desigualdad entre 1999 y 2008 . En estos años, el índice de Gini solo se redujo un 4,3\%, el índice de Theil un $8,1 \%$ y el índice 10/40 un 15,3\%. La región sur, en cambio, mostró los mejores resultados, ya que no solo registró los niveles más bajos de desigualdad a lo largo del período, sino que también logró el retroceso más pronunciado de la desigualdad (12\% de disminución del índice de Gini, 22,8\% del índice de Theil y 32,4\% del índice 10/40).

En cuanto a las variables económicas, suele considerarse que la apertura comercial es relevante para el análisis de la desigualdad de los ingresos (Rodrik, 1997; Easterly, 2005; Nissanke y Thorbecke, 2006). Una razón de esa relevancia estriba en que la relación entre la distribución de los ingresos y la apertura comercial depende en parte de la medida en que se usen los factores de producción para los principales productos de un país. Las diferencias entre países pueden verse aumentadas 
GRÁFICO

Brasil (regiones): indicadores de desigualdad, 1999-2008

A. Índice de Gini

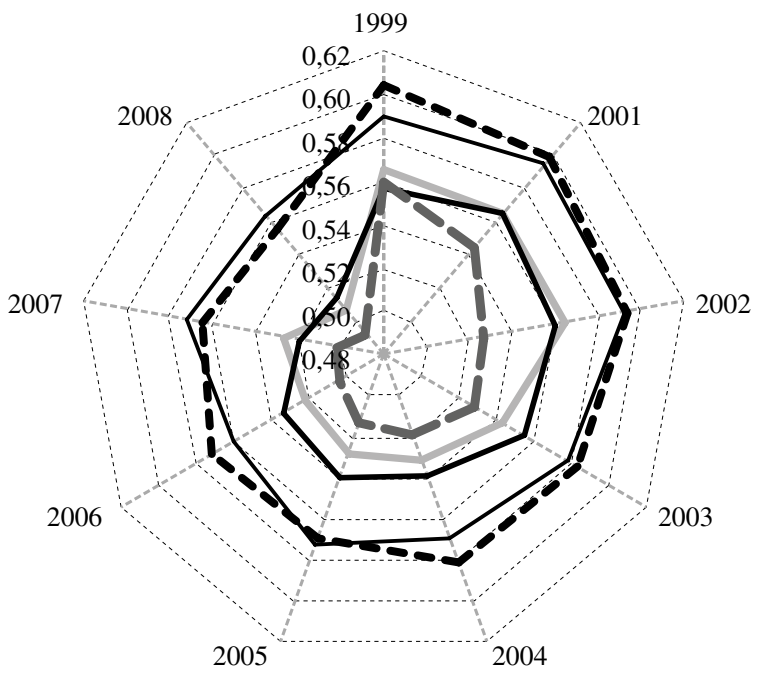

B. Índice de Theil

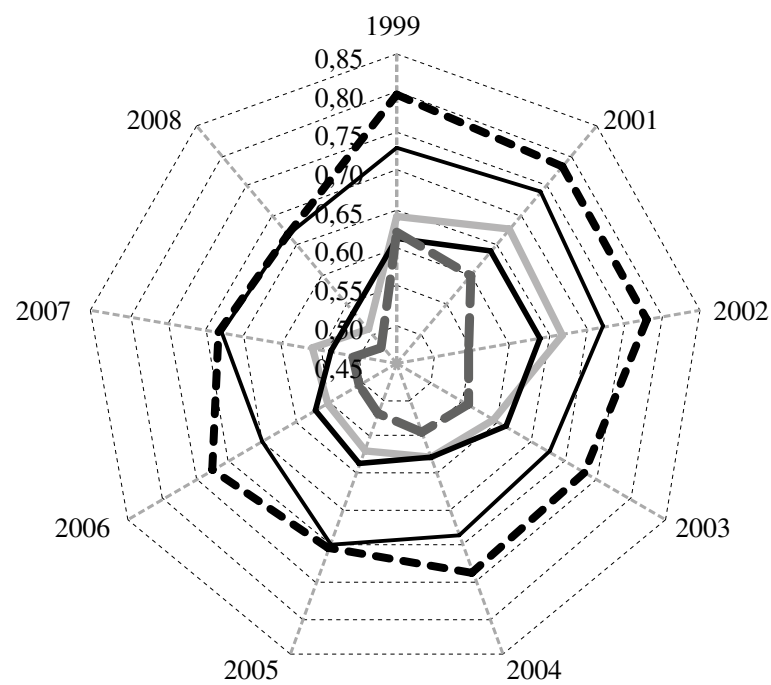

C. Índice $10 / 40$

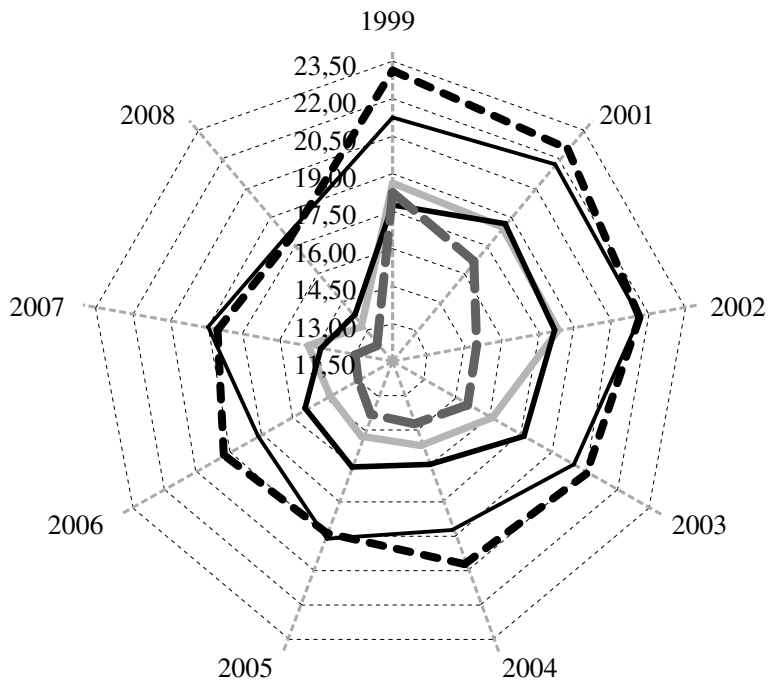

Norte

- - Nordeste

Centro-oeste

Sudeste

$\infty \cdot$ Sur

Fuente: Instituto Brasileño de Geografía y Estadística (IBGE). 
por las distintas legislaciones fiscales y laborales, pero este efecto resulta insignificante para el análisis de los estados de la República Federativa del Brasil, ya que todos están sometidos a una misma legislación. Por lo tanto, en este estudio se utiliza el siguiente indicador de apertura comercial entre los estados y los países extranjeros, basado en los datos del Ministerio para el Desarrollo, la Industria y el Comercio Exterior (MDIC) y del Banco Central del Brasil: OPEN = (importaciones totales + exportaciones totales)/PIB.

Como se muestra en el gráfico 2, la región centro-oeste obtuvo, de nuevo, los peores resultados durante el período, pero su apertura comercial mejoró considerablemente después de 2002 y adelantó a la región nordeste en 2008. La región sudeste registró los datos más estables de todas las regiones y mantuvo de forma permanente una posición intermedia. La región norte mostró la mayor apertura comercial, si bien la región sur se fue acercando a su nivel.

Otra variable fundamental para el análisis de la desigualdad de los ingresos es el desarrollo tecnológico (Acemoglu y Newman, 2002; Madsen, 2007; Fang, Huang y Wang, 2008). En este análisis se utiliza como aproximación al desarrollo tecnológico la proporción entre las patentes concedidas en cada estado y el número total de patentes otorgadas en el Brasil cada año (PAT), sobre la base de los datos del Instituto Nacional de la Propiedad Industrial ${ }^{3}$. Esta aproximación permite observar el crecimiento tecnológico medio del país. Sin embargo, debido a que la variable es una proporción, podría incluso disminuir si el número total de patentes aumentase en un estado concreto, en caso de que la tasa de crecimiento del estado fuera inferior a la tasa de crecimiento media del país en conjunto. Para facilitar la interpretación de los resultados, se normaliza la aproximación a un intervalo de 0 a 1 . En el gráfico 3 se observa que gran parte del desarrollo tecnológico se concentró en la región sudeste, mientras que las regiones norte y medio-oeste tuvieron un desarrollo insignificante. Los valores de este indicador no cambiaron de forma significativa entre las regiones durante el período considerado.

Otra variable relevante para la desigualdad de los ingresos es el desarrollo financiero (Greenwood y Jovanovic, 1990; Galor y Zeira, 1993; Aghion y Bolton, 1997; Claessens y Perotti, 2005). Como señala Kumar (2005), las personas con acceso a servicios financieros pueden protegerse de períodos con escasos ingresos o de fluctuaciones inesperadas de los ingresos, mejorando así la asignación de recursos. Además, un sistema

3 En 2007 y 2008 se han restado las patentes emitidas desde el extranjero de las atribuidas a Río de Janeiro.

GRÁFICO 2

Brasil (regiones): apertura comercial (OPEN), 1999-2008

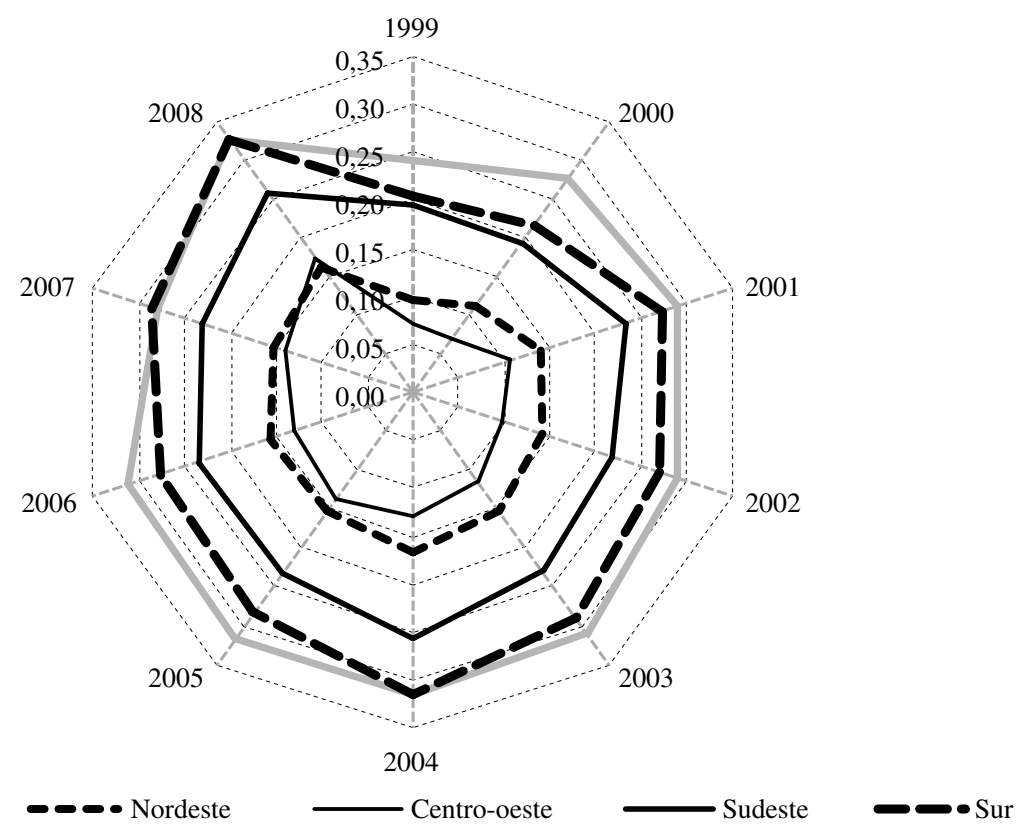

Fuente: Ministerio para el Desarrollo, la Industria y el Comercio Exterior (MDIC) y Banco Central del Brasil.

Nota: la variable OPEN es la suma de importaciones y exportaciones, dividida por el producto interno bruto (PIB). 


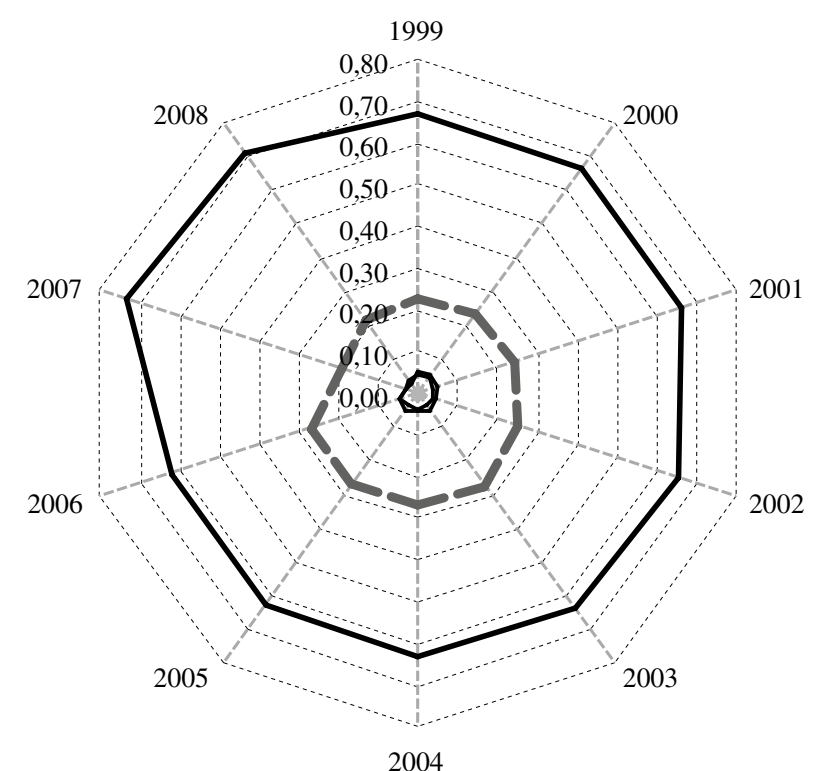

2004

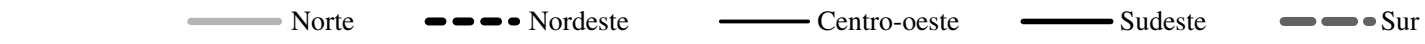

Fuente: Instituto Nacional de la Propiedad Industrial.

Nota: la variable PAT es la proporción entre las patentes concedidas en cada estado y el número total de patentes otorgadas en el Brasil.

financiero desarrollado permite que las personas en condiciones de pobreza tengan acceso a los servicios financieros. En el Brasil, casi todos los clientes del sistema bancario utilizan libretas de ahorros ${ }^{4}$. Por lo tanto, como aproximación al desarrollo financiero (FD) se utiliza el saldo total del ahorro en los diferentes estados en diciembre de cada año, dividido por el PIB de los estados (en cifras reales del año 2000) sobre la base de las estadísticas bancarias proporcionadas por el Banco Central del Brasil. En el gráfico 4 se evidencia que este indicador disminuyó en todas las regiones durante los primeros años de la muestra. La región sudeste obtuvo en general los mejores resultados, pero las regiones sur y nordeste experimentaron una notable recuperación hacia finales del período.

Entre 1999 y 2008, el Gobierno del Brasil puso en práctica varias iniciativas que, en combinación con un buen entorno macroeconómico, repercutieron significativamente en los niveles de desigualdad y en el mercado laboral. Algunas de las principales medidas fueron los aumentos reales sucesivos del salario mínimo y la implementación del programa Bolsa Família de

4 Según Kumar (2005), el 97\% de todos los clientes del sistema bancario en el Brasil conservan su dinero en depósitos de ahorros. transferencias condicionales. En relación con el salario mínimo, una ley federal fija el límite inferior y cada estado puede establecer su propio salario mínimo en ese límite o por sobre este. Los aumentos del salario mínimo no solo afectan a los trabajadores que reciben ese salario, sino que también se difunden en un intervalo en su entorno tanto en el sector formal de la economía como en el informal (sin acrecentar la tasa de desempleo) (Lemos, 2009). Además, los efectos de un aumento no se limitan a los salarios, también influyen en las prestaciones de jubilación y en los seguros de desempleo ${ }^{5}$. Por lo tanto, un cambio en esta variable tiene una repercusión relevante en los ingresos de la población. Para determinar este impacto, se considera la variación anual del salario mínimo (MW) en cada estado en cifras reales del año 2000. En el gráfico 5 se advierte la variación del límite inferior del salario mínimo, definido por el gobierno federal, y del salario mínimo real en los estados en que se estableció un valor diferente. En general, después de que un estado establece un salario mínimo por sobre el límite inferior nacional, dicho salario suele seguir el comportamiento del salario mínimo nacional.

\footnotetext{
5 Según el Ministerio del Trabajo y Empleo, en 2003 estas categorías tuvieron un efecto directo en más del $8,4 \%$ de la población.
} 
GRÁFICO 4

Brasil (regiones): desarrollo financiero (FD), 1999-2008

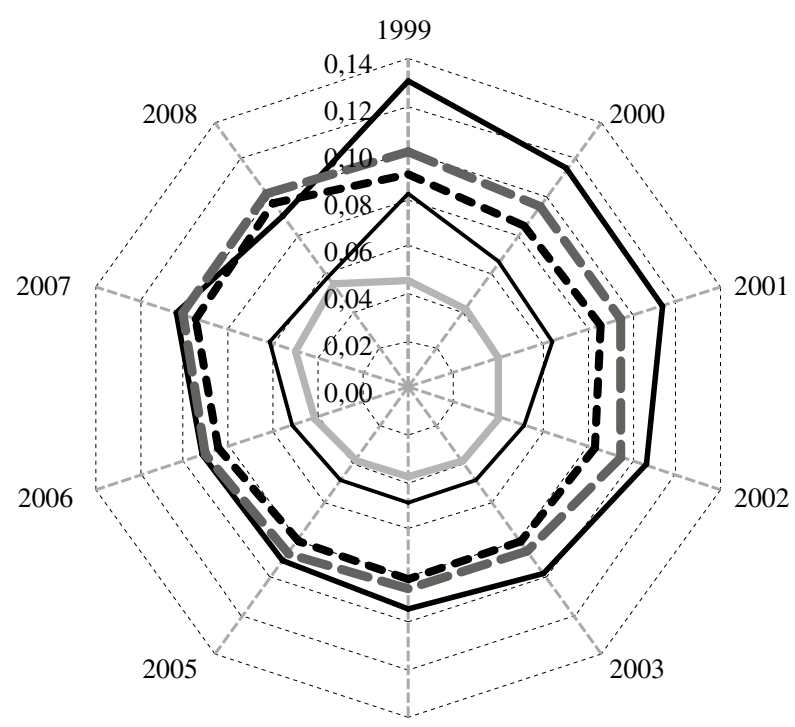

2004

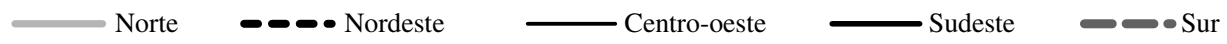

Fuente: Banco Central del Brasil.

Nota: la variable FD consiste en los ahorros totales divididos por el producto interno bruto (PIB).

GRÁFICO 5

Brasil (cuatro estados): variación anual del salario mínimo (MW), 1999-2008

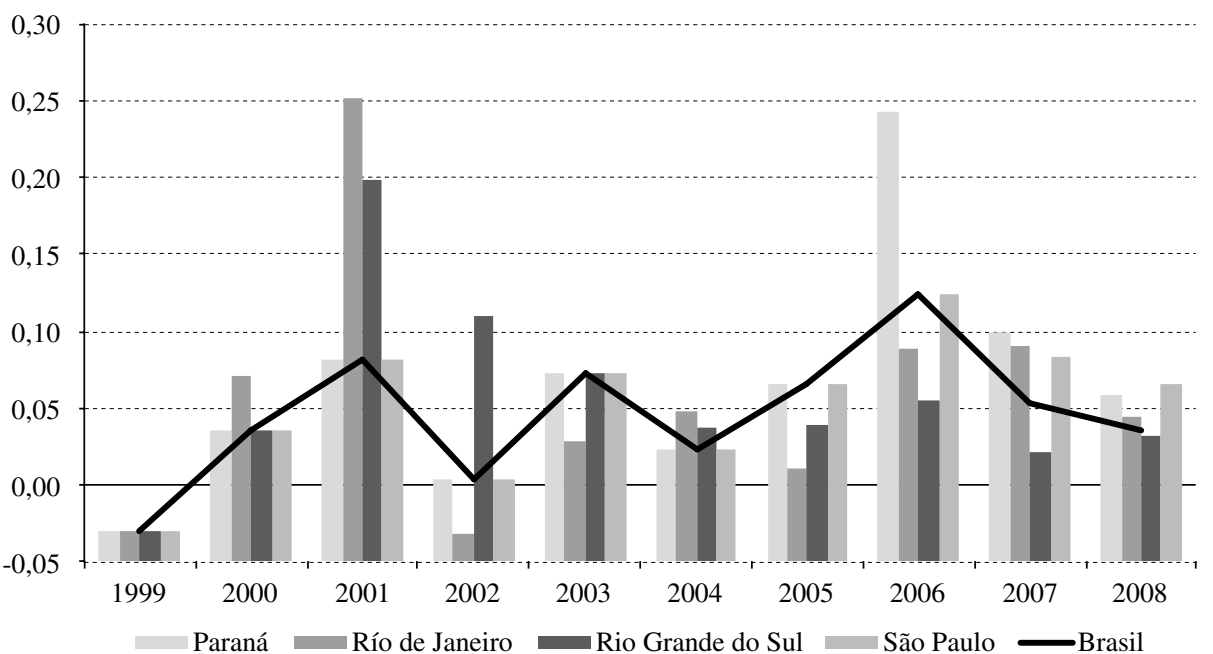

Fuente: Ministerio del Trabajo y Empleo, y leyes estatales.

Nota: la variable mw consiste en la variación anual del salario mínimo, dividida por este último. 
El desempleo es otra variable fundamental para el análisis de la desigualdad. En consecuencia, se incluye la tasa de desempleo por estados (UNE), tanto en el sector formal como en el informal, utilizando los datos del Instituto de Investigaciones Económicas Aplicadas (IPEA). En el gráfico 6 se aprecia que todas las regiones registraron un descenso de la tasa de desempleo en el período considerado, pero con diferencias significativas. Las peores cifras fueron las de la tasa de desempleo en la región nordeste, que solo se redujo un 3,5\% en 10 años. En cambio, en la región sur dicha tasa disminuyó un 38\%. La región sudeste mantuvo la mayor tasa de desempleo del país, pero terminó el período cerca de los niveles observados en la región nordeste y en la centro-oeste (con una reducción del 27,4\%, el segundo mejor resultado del país).

El programa Bolsa Família de transferencias condicionales, iniciado en 2004, fue una pieza clave de las políticas contra la pobreza del Gobierno del Brasil durante el período analizado. En virtud de este programa, las familias pobres con hijos reciben un promedio de 70 reales (unos 40 dólares) en transferencias directas, con la condición de que se comprometan a mantener a sus hijos en la escuela y a llevarlos a revisiones médicas periódicas. Esta iniciativa social del Ministerio de Desarrollo Social y Lucha contra el Hambre alcanzó a una gran parte de la población con bajos ingresos del país (más de 46 millones de personas).
El programa Bolsa Família solo concierne a aquella parte de la población cuyos ingresos per cápita son inferiores a 140 reales al mes. Por lo tanto, se puede usar la proporción entre los beneficiarios de Bolsa Família y la población total para determinar el efecto real del programa en la población del país. En cambio, otras formas posibles de medir ese efecto, como la proporción entre los beneficiarios de Bolsa Família y la población pobre, contienen ciertas distorsiones. En regiones donde existe un menor número de pobres, el aumento de los beneficiarios del programa tiene un enorme efecto en la proporción entre los beneficiarios y la población pobre, pero esta proporción no refleja la repercusión en la distribución de los ingresos de la población total. Por consiguiente, para analizar el efecto en la desigualdad, en este estudio se considera la proporción entre el número de beneficiarios de Bolsa Família y la población total de cada estado (BF), utilizando datos del Instituto de Investigaciones Económicas Aplicadas (IPEA).

Como se observa en el gráfico 7, esta variable creció en todas las regiones durante los primeros tres años del programa. Puesto que Bolsa Família está dirigido a las familias pobres, no resulta sorprendente que la región sur experimentase el crecimiento más bajo durante el período (18\%). Por el contrario, en el norte se produjo un aumento del $93,5 \%$ y la región nordeste tuvo el nivel más alto de $\mathrm{BF}$.

GRÁFICO 6

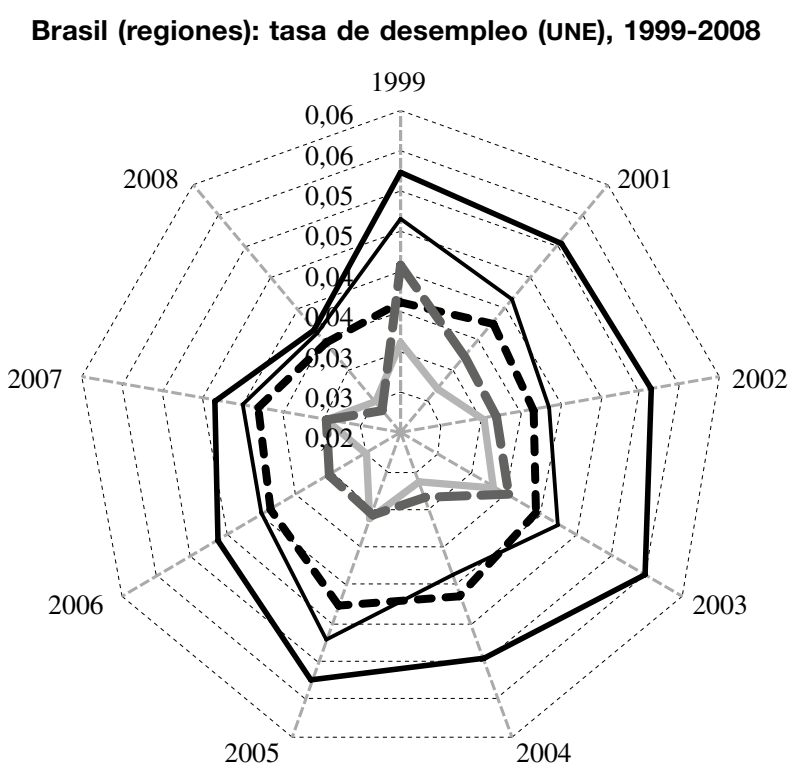

Norte - - - Nordeste Centro-oeste Sudeste $\longrightarrow$ - Sur

Fuente: Instituto de Investigaciones Económicas Aplicadas (IPEA).

Nota: la variable UNE consiste en la tasa de desempleo (formal e informal). 


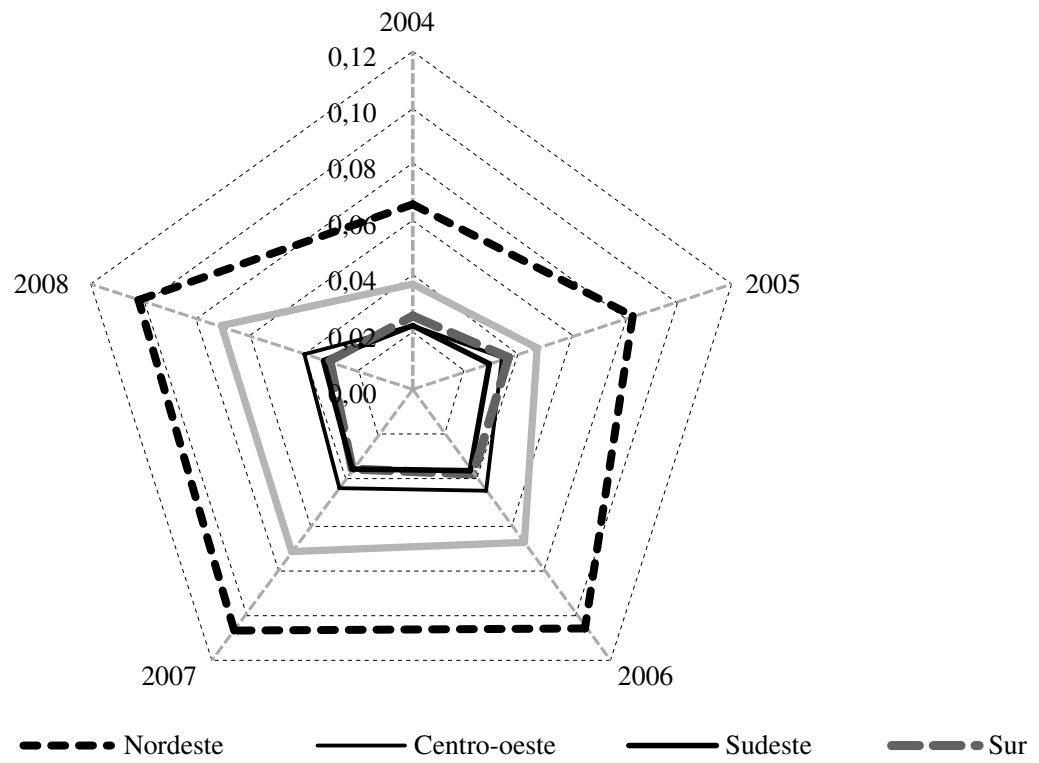

Fuente: Ministerio de Desarrollo Social y Lucha contra el Hambre.

Nota: la variable BF consiste en la proporción entre el número de beneficiarios de Bolsa Família y la población total.

En este análisis se han incluido algunas variables políticas, para tener en cuenta la posibilidad de que los factores políticos influyan en la distribución de los ingresos. Según Levitt y Poterba (1999) y Rupasingha y Goetz (2007), existe una correlación entre un sistema democrático y un nivel inferior de pobreza. En consecuencia, en el estudio se consideran los cambios en el poder político en los estados del Brasil. Entre 1999 y 2008, hubo tres mandatos electorales en los estados (1999-2002, 2003-2006 y 2007-2010). El indicador de cambio político (PC) es una variable ficticia cuyo valor es igual a uno (1) si el partido del gobierno anterior era diferente del partido del gobierno actual y, en caso contrario, es igual a cero (0).

En el cuadro 1 se muestra que es habitual que un partido político permanezca en el poder durante dos o más mandatos consecutivos. Solo en seis estados no se produjo esta tendencia. En particular, en los estados del sur se produjeron dos (Paraná y Santa Catarina) o tres (Rio Grande do Sul) cambios políticos a lo largo del período. Puesto que la región del sur tiene los mejores índices relativos a la desigualdad de los ingresos, estos

CUADRO 1

Brasil (regiones): episodios de cambio político (PC), 1999-2008

\begin{tabular}{ll}
\hline No de cambios & Estados y Distrito Federal \\
\hline 0 & São Paulo (SE) \\
1 & Acre (N), Amapá (N), Bahia (NE), Ceará (NE), Maranhão (NE), Pará (N), Paraíba (NE), Piauí (NE), Rio Grande do \\
& Norte (NE) \\
& Alagoas (NE), Distrito Federal (Mw), Goiás (Mw), Minas Gerais (SE), Mato Grosso (Mw), Pernambuco (NE), \\
& Paraná (s), Santa Catarina (s), Sergipe (NE), Tocantins (Mw) \\
& Amazonas (N), Espírito Santo (SE), Río de Janeiro (SE), Rondônia (N), Roraima (N), Rio Grande do Sul (s)
\end{tabular}

Fuente: Instituto de Investigaciones Económicas Aplicadas (IPEA).

Nota: $\mathrm{SE}=$ Sudeste $; \mathrm{s}=$ Sur $; \mathrm{N}=$ Norte $; \mathrm{NE}=$ Nordeste $; \mathrm{MW}=$ Centro-oeste. 
datos podrían corroborar la idea de que la democracia reduce la desigualdad. Sin embargo, esto no se puede confirmar con los resultados de las demás regiones.

Para evaluar la repercusión del gasto social de un estado (es decir, los gastos en servicios sociales y en la seguridad social), se ha calculado la proporción entre las transferencias directas a los ciudadanos y el PIB del estado (soc) utilizando los datos del Ministerio de Hacienda. Salvo la región nordeste, todas las regiones registraron una disminución del gasto social a lo largo del período (véase el gráfico 8). Una de las razones que explican esta tendencia es la Ley de Responsabilidad Fiscal aprobada por el Congreso Nacional del Brasil en el año 2000, que limita los gastos en personal de los estados al 60\% de sus ingresos corrientes netos.

Finalmente, se analiza el impacto de la corrupción en la desigualdad de los ingresos (Gupta, Davoodi y Alonso-Terme, 2002; Alt y Lassen, 2010). Según el índice de percepción de la corrupción publicado por Transparencia Internacional, el índice del Brasil empeoró considerablemente entre 1999 y 2008, y el país pasó del puesto 45 al 80 en la lista de países con menor corrupción. En este estudio se utiliza la variación del índice de corrupción (CORR) creado por Boll (2010) y basado en el promedio ponderado de algunas variables socioeconómicas (población y PIB del estado $=0,33$ ) y el número de procesos considerados como ilegales por el Tribunal de Cuentas del Brasil $(0,66)$, según la ley de presupuesto anual. El índice puede tomar valores entre cero (0) (menor corrupción) y uno (1) (mayor corrupción). En el gráfico 9 se representa el promedio de corrupción de cada estado a lo largo del período. Los tres índices más altos correspondieron a la región nordeste (Maranhão, Piauí y Bahia), mientras que los dos más bajos estuvieron en el sur (Rio Grande do Sul y Santa Catarina).
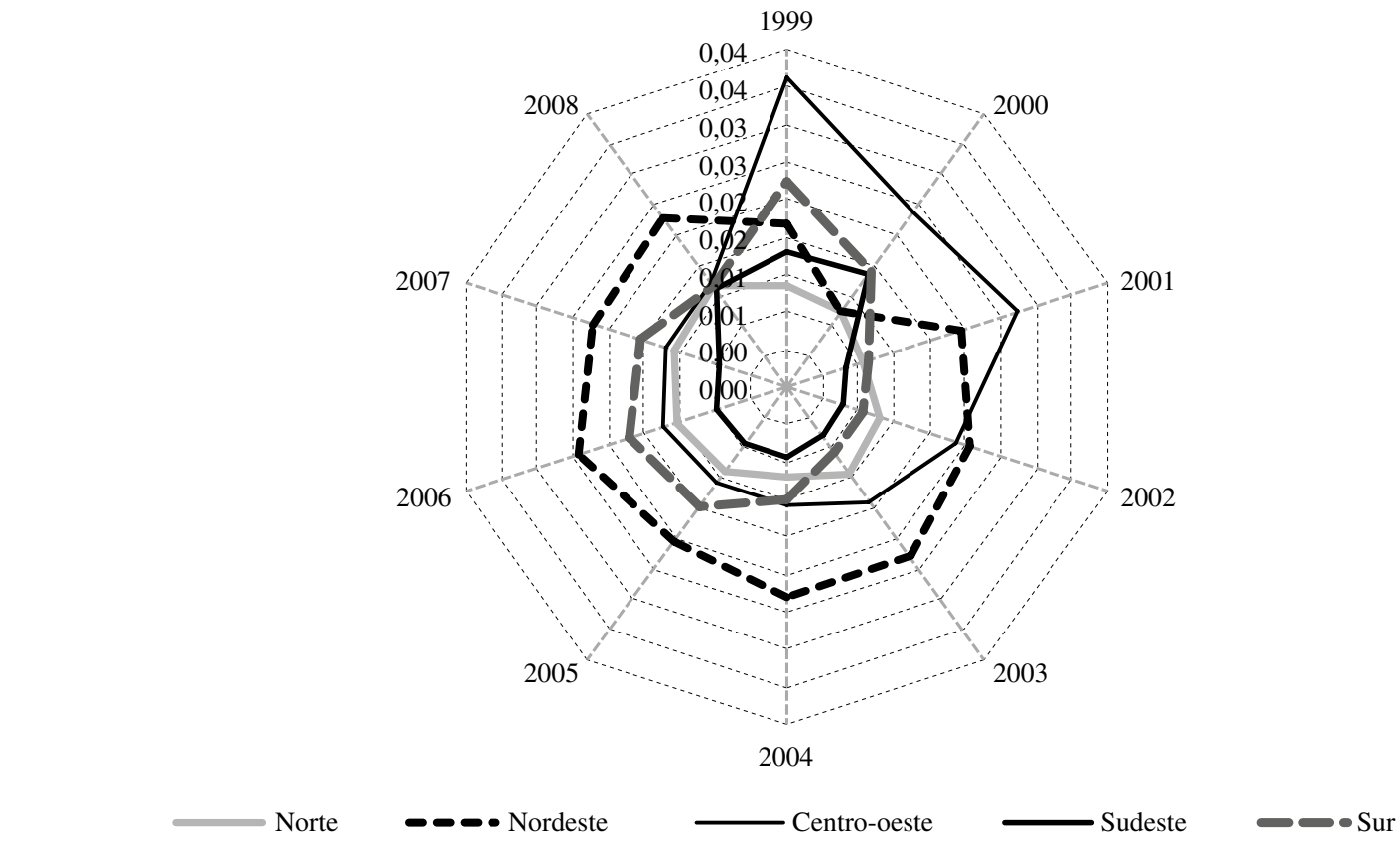

Fuente: Ministerio de Hacienda.

Nota: la variable soc consiste en la proporción entre las transferencias directas a los ciudadanos y el producto interno bruto (PIB). 
GRÁFICO 9

Brasil: promedio de corrupción en los estados y en el Distrito Federal (CORR), 1999-2008

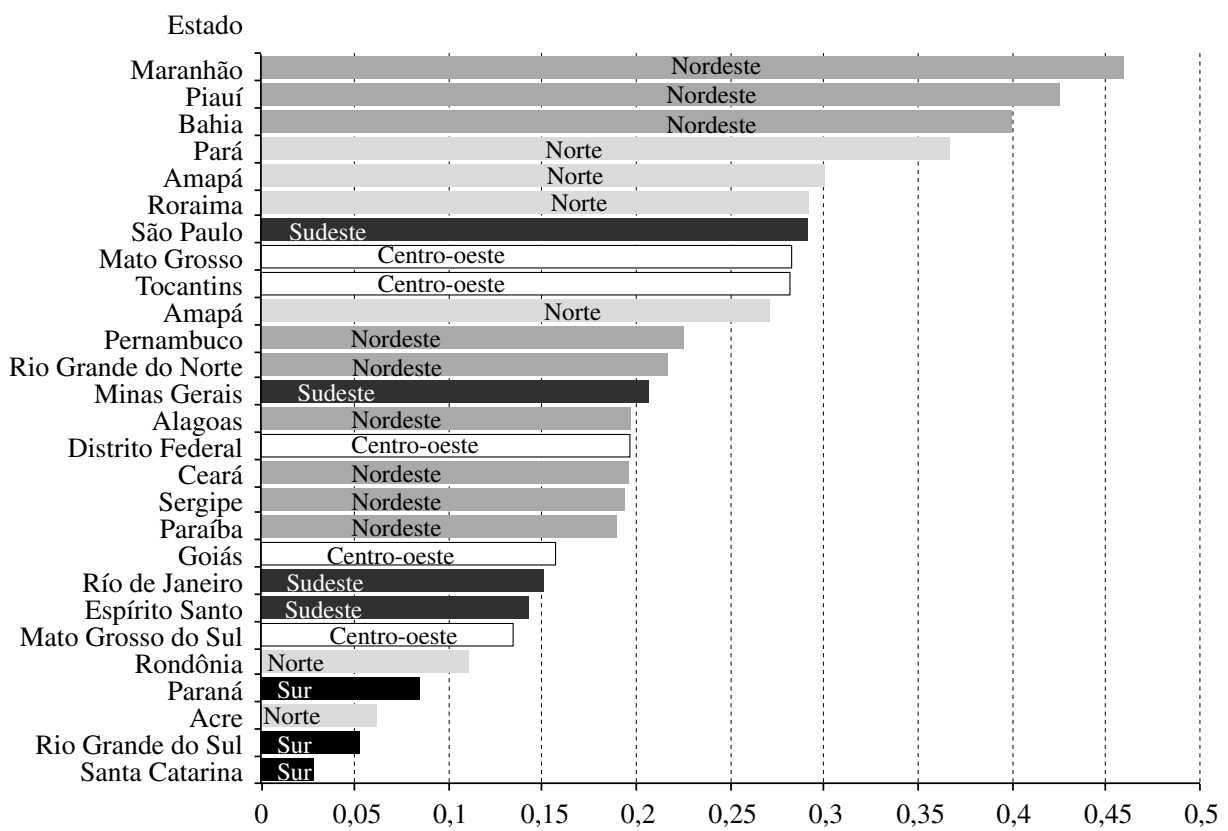

Fuente: J.L.S. Boll, "A corrupção governamental no Brasil: construção de indicadores e análise da sua incidência relativa nos estados brasileiros”, Brasilia, 2010 [en línea] http://www.cgu.gov.br/concursos/Arquivos/5_ConcursoMonografias/MH-profissionais-jose-luisserafini-boll.pdf.

Nota: el eje horizontal corresponde al promedio del índice de corrupción (CORR).

\section{III}

\section{Datos empíricos}

Las variables presentadas en la sección anterior proporcionan un total de 270 valores (con frecuencia anual) para los 26 estados del Brasil, más el Distrito Federal, durante el período comprendido entre 1999 y 2008. En esta sección se presentan los datos empíricos, utilizando una estimación mediante el método generalizado de momentos (MGM) para el análisis de datos de panel (en el cuadro 2 se muestra la estadística descriptiva). Según Arellano y Bond (1991), una ventaja de utilizar el MGM para datos de panel dinámicos consiste en que, además de eliminar los efectos no observables sobre las regresiones, las estimaciones son fiables aunque se omitan variables. En particular, el uso de variables instrumentales permite realizar una estimación más coherente de los parámetros, incluso en caso de endogeneidad de las variables explicativas y en presencia de errores de medida (Bond, Hoeffler y Temple, 2001).
Con los modelos econométricos tradicionales se supone que no existe una correlación entre el término de error y sus estimadores. Cuando existe una correlación entre los estimadores y el término de error, hay un problema de endogeneidad y los resultados de la regresión son inconsistentes. Wooldridge (2001) señala tres posibilidades de que existan variables endógenas: variables omitidas, error de medida y problemas de simultaneidad. El modelo empírico elaborado en este estudio está sujeto a esos problemas. Por ejemplo, la apertura comercial puede reducir la desigualdad de los ingresos, pero esa reducción, a su vez, puede provocar un aumento de las importaciones y modificar así la apertura comercial.

Una solución general para el problema de la endogeneidad consiste en el uso de variables instrumentales. Los modelos en que se utiliza el MGM 
CUADRO 2

Valores estadísticos descriptivos

\begin{tabular}{|c|c|c|c|c|c|}
\hline Variable & Promedio & Mediana & Máximo & Mínimo & Desviación estándar \\
\hline Índice de Gini & 0,5538 & 0,5545 & 0,6545 & 0,4486 & 0,0375 \\
\hline Índice de Theil & 0,1788 & 0,1739 & 0,3266 & 0,0954 & 0,0412 \\
\hline Índice 10/40 & 0,6376 & 0,6262 & 1,0369 & 0,3875 & 0,1106 \\
\hline OPEN & 0,1707 & 0,1270 & 0,6051 & 0,0023 & 0,1446 \\
\hline PAT & 0,0370 & 0,0043 & 0,6000 & 0,0000 & 0,0940 \\
\hline FD & 0,0751 & 0,0795 & 0,1419 & 0,0282 & 0,0264 \\
\hline MW & 0,0493 & 0,0535 & 0,2511 & $-0,1411$ & 0,0543 \\
\hline UNE & 0,0419 & 0,0406 & 0,0801 & 0,0180 & 0,0126 \\
\hline $\mathrm{BF}$ & 0,0338 & 0,0237 & 0,1208 & 0,0000 & 0,0375 \\
\hline SOC & 0,0196 & 0,0195 & 0,0737 & 0,0017 & 0,0103 \\
\hline CORR & 1,0955 & $-0,0074$ & 40,5000 & $-1,0000$ & 4,1627 \\
\hline
\end{tabular}

Fuente: elaboración propia.

permiten usar instrumentos secuencialmente exógenos, evitando así el problema de endogeneidad. Arellano y Bond (1991) propusieron el uso de un estimador del MGM en primeras diferencias para datos de panel como forma de eliminar los efectos no observables. Sin embargo, Alonso-Borrego y Arellano (1998) y Blundell y Bond (1998) muestran que el estimador del MGM en primeras diferencias está sesgado para muestras grandes y pequeñas y su precisión es escasa. Además, el uso de retrasos puede crear instrumentos débiles (Staiger y Stock, 1997). Por lo tanto, Blundell y Bond (1998) recomiendan utilizar la estimación de sistema del MGM para datos de panel en lugar del MGM en primeras diferencias. Según la propuesta de Arellano y Bover (1995) y Blundell y Bond (1998), en este estudio se combinan las regresiones en niveles y en primeras diferencias (véase Bond, Hoeffler y Temple, 2001).

Para comprobar los instrumentos de los modelos, se realizó la prueba de Sargan de restricciones de sobreidentificación, como sugiere Arellano (2003). Por otra parte, la matriz de covarianza consistente con la heteroscedasticidad de White se aplicó a las regresiones, así como la prueba de correlación serial de segundo orden (m2) propuesta por Arellano y Bond (1991). No se realizaron pruebas de raíz unitaria, ya que una premisa de los modelos de sistema del MGM es la ausencia de correlación de la primera diferencia de los regresores endógenos.

Para encontrar datos empíricos sobre el efecto de las variables descritas en la sección anterior sobre los índices de desigualdad (el índice de Gini, el índice de Theil y el índice 10/40), se han considerado dos series de modelos de sistema del MGM utilizando datos de panel. En la primera serie - ecuaciones (1) a (4) — se incluyen las variables convencionales (OPEN, PAT y FD) y las variables socioeconómicas (MW, UNE y BF). En la segunda serie —ecuaciones (5) a (7) — también se consideran OPEN, PAT y FD, pero incluyendo variables sujetas a algún tipo de interferencia política (PC, SOC y CORR). Por lo tanto,

$$
\begin{gathered}
X_{i, t}=\beta_{0} X_{i, t-1}+\beta_{1} O P E N_{i, t}+\beta_{2} P A T_{i, t}+ \\
\beta_{3} F D_{i, t}+\mu_{i, t}^{X, 1} ;
\end{gathered}
$$

$$
\begin{gathered}
X_{i, t}=\beta_{4} X_{i, t-1}+\beta_{5} O P E N_{i, t}+\beta_{6} P A T_{i, t}+ \\
\beta_{7} F D_{i, t}+\beta_{8} M W_{i, t}+\mu_{i, t}^{X, 2} ;
\end{gathered}
$$

$$
\begin{aligned}
X_{i, t}= & \beta_{9} X_{i, t-1}+\beta_{10} O P E N_{i, t}+\beta_{11} P A T_{i, t}+ \\
& \beta_{12} F D_{i, t}+\beta_{13} U N E_{i, t}+\mu_{i, t}^{X, 3} ;
\end{aligned}
$$

$$
\begin{gathered}
X_{i, t}=\beta_{14} X_{i, t-1}+\beta_{15} O P E N_{i, t}+\beta_{16} P A T_{i, t}+ \\
\beta_{17} F D_{i, t}+\beta_{18} B F_{i, t}+\mu_{i, t}^{X, 4} ;
\end{gathered}
$$

$$
\begin{gathered}
X_{i, t}=\beta_{19} X_{i, t-1}+\beta_{20} O P E N_{i, t}+\beta_{21} P A T_{i, t}+ \\
\beta_{22} F D_{i, t}+\beta_{23} P C_{i, t}+\mu_{i, t}^{X, 5} ;
\end{gathered}
$$$$
\begin{gathered}
X_{i, t}=\beta_{24} X_{i, t-1}+\beta_{25} \operatorname{OPEN}_{i, t}+\beta_{26} P A T_{i, t}+ \\
\beta_{27} F D_{i, t}+\beta_{28} \operatorname{SOC}_{i, t}+\mu_{i, t}^{X, 6} ;
\end{gathered}
$$$$
X_{i, t}=\beta_{29} X_{i, t-1}+\beta_{30} \text { OPEN }_{i, t}+\beta_{31} P A T_{i, t}+
$$$$
\beta_{32} F D_{i, t}+\beta_{33} \operatorname{CORR}_{i, t}+\mu_{i, t}^{X, 7}
$$

donde $\mu_{i, t}^{X} \sim N\left(0, \sigma^{2}\right)$, y $X$ es el índice de desigualdad (el índice de Gini, el índice de Theil o el índice 10/40). 
En los cuadros 3, 4 y 5 se evidencian los resultados de la estimación para los modelos. Todas las regresiones asumen la hipótesis nula en las pruebas de Sargan, de modo que las restricciones de sobreidentificación son válidas. Además, mediante las pruebas de autocorrelación serial se rechaza la hipótesis de su presencia.

Independientemente del indicador de desigualdad utilizado en las estimaciones, el coeficiente de la variable OPEN es negativo y estadísticamente significativo en todas las especificaciones. Este resultado es coherente con la idea de que el aumento de la apertura comercial constituye un potente mecanismo para lograr que disminuya la desigualdad de los ingresos. Los coeficientes de PAT y FD también son negativos, aunque no siempre estadísticamente significativos, de manera que puede deducirse que el desarrollo tecnológico y financiero contribuye a reducir la desigualdad de los ingresos.

Los coeficientes de MW son negativos y estadísticamente significativos en las tres especificaciones. Este resultado indica que la política gubernamental de llevar a cabo aumentos reales del salario mínimo podría ser un mecanismo apropiado para combatir la desigualdad de los ingresos. Todas las especificaciones muestran que el coeficiente de UNE es estadísticamente

CUADRO 3

El índice de desigualdad de Gini: estimador de sistema del MGM

\begin{tabular}{|c|c|c|c|c|c|c|c|}
\hline Variable & (1) & (2) & (3) & (4) & (5) & (6) & (7) \\
\hline $\operatorname{Gini}_{i, t-1}$ & $\begin{array}{c}0,3661^{\mathrm{b}} \\
(0,1542) \\
{[2,3728]}\end{array}$ & $\begin{array}{c}0,2312^{\mathrm{c}} \\
(0,1328) \\
{[1,7409]}\end{array}$ & $\begin{array}{c}0,0311 \\
(0,2349) \\
{[0,1323]}\end{array}$ & $\begin{array}{c}0,1436 \\
(0,1679) \\
{[0,8557]}\end{array}$ & $\begin{array}{c}0,3959^{\mathrm{b}} \\
(0,1888) \\
{[-2,0970]}\end{array}$ & $\begin{array}{c}0,2413^{\mathrm{c}} \\
(0,1275) \\
{[1,8922]}\end{array}$ & $\begin{array}{c}0,6734^{\mathrm{a}} \\
(0,1583) \\
{[4,2536]}\end{array}$ \\
\hline $\operatorname{OPEN}_{i, t-1}$ & $\begin{array}{c}-0,3450^{\mathrm{a}} \\
(0,0945) \\
{[-3,6483]}\end{array}$ & $\begin{array}{c}-0,3036^{\mathrm{a}} \\
(0,0970) \\
{[-3,1281]}\end{array}$ & $\begin{array}{c}-0,2307^{c} \\
(0,1377) \\
{[-1,6747]}\end{array}$ & $\begin{array}{c}-0,2586^{b} \\
(0,0818) \\
{[-3,1578]}\end{array}$ & $\begin{array}{c}-0,2899^{a} \\
(0,1055) \\
{[-2,7472]}\end{array}$ & $\begin{array}{c}-0,2735^{a} \\
(0,0801) \\
{[-3,4104]}\end{array}$ & $\begin{array}{r}-0,2812^{b} \\
(0,1083) \\
{[-2,5960]}\end{array}$ \\
\hline $\mathrm{PAT}_{i, t-1}$ & $\begin{array}{c}-0,1815^{c} \\
(0,0998) \\
{[-1,8189]}\end{array}$ & $\begin{array}{c}-0,2217^{b} \\
(0,1053) \\
{[-2,1061]}\end{array}$ & $\begin{array}{c}-0,1132 \\
(0,0861) \\
{[-1,3146]}\end{array}$ & $\begin{array}{c}-0,1983^{a} \\
(0,0704) \\
{[-2,8172]}\end{array}$ & $\begin{array}{c}-0,2195 \\
(0,1685) \\
{[-1,3027]}\end{array}$ & $\begin{array}{c}-0,2194^{b} \\
(0,0862) \\
{[-2,5435]}\end{array}$ & $\begin{array}{c}-0,1286 \\
(0,1211) \\
{[-1,0614]}\end{array}$ \\
\hline $\mathrm{FD}_{i, t-1}$ & $\begin{array}{c}-0,5812^{c} \\
(0,3196) \\
{[-1,8184]}\end{array}$ & $\begin{array}{c}-0,6857^{b} \\
(0,3032) \\
{[-2,2617]}\end{array}$ & $\begin{array}{c}-0,6188^{b} \\
(0,2503) \\
{[-2,4715]}\end{array}$ & $\begin{array}{c}-0,3778^{c} \\
(0,2276) \\
{[-1,6603]}\end{array}$ & $\begin{array}{c}-0,6792^{c} \\
(0,3718) \\
{[-1,8265]}\end{array}$ & $\begin{array}{c}-0,4910^{c} \\
(0,2560) \\
{[-1,9179]}\end{array}$ & $\begin{array}{c}-0,2623 \\
(0,3304) \\
{[-0,7940]}\end{array}$ \\
\hline $\mathrm{MW}_{i, t-1}$ & & $\begin{array}{c}-0,0641^{\mathrm{c}} \\
(0,0383) \\
{[-1,6697]}\end{array}$ & & & & & \\
\hline $\mathrm{UNE}_{i, t-1}$ & & & $\begin{array}{l}1,1295^{\mathrm{c}} \\
(0,6403) \\
{[1,7638]}\end{array}$ & & & & \\
\hline $\mathrm{BF}_{i, t-1}$ & & & & $\begin{array}{c}-0,2502^{\mathrm{a}} \\
(0,0899) \\
{[-2,7830]}\end{array}$ & & & \\
\hline $\mathrm{PC}_{i, t-1}$ & & & & & $\begin{array}{c}0,0197^{\mathrm{c}} \\
(0,0114) \\
{[1,7350]}\end{array}$ & & \\
\hline $\operatorname{SOC}_{i, t-1}$ & & & & & & $\begin{array}{c}1,3791^{\mathrm{b}} \\
(0,5954) \\
{[2,3162]}\end{array}$ & \\
\hline $\mathrm{CORR}_{i, t-1}$ & & & & & & & $\begin{array}{c}0,0011^{\mathrm{a}} \\
(0,0004) \\
{[2,8408]}\end{array}$ \\
\hline Estadístico $J$ & $\begin{array}{l}15,7519 \\
p>0,35\end{array}$ & $\begin{array}{l}16,8766 \\
p>0,45\end{array}$ & $\begin{array}{c}4,3807 \\
\mathrm{p}>0,95\end{array}$ & $\begin{array}{l}17,8650 \\
p>0,45\end{array}$ & $\begin{array}{c}8,1331 \\
\mathrm{p}>0,70\end{array}$ & $\begin{array}{l}23,1068 \\
p>0,25\end{array}$ & $\begin{array}{l}14,8607 \\
p>0,35\end{array}$ \\
\hline $\mathrm{m}^{2}$ & $-0,0796$ & $-0,1286$ & 0,0789 & $-0,1100$ & $-0,0444$ & $-0,1144$ & $-0,0325$ \\
\hline Valor $p$ & 0,6703 & 0,4619 & 0,7726 & 0,5058 & 0,7956 & 0,4712 & 0,8362 \\
\hline Instrumentos & 20 & 22 & 21 & 23 & 16 & 25 & 19 \\
\hline
\end{tabular}

Fuente: elaboración propia.

Nota: los errores estándar están entre paréntesis y el valor $t$ está entre corchetes.

MGM: método generalizado de momentos.

a Significativo a un nivel del $1 \%$.

b Significativo a un nivel del $5 \%$.

c Significativo a un nivel del $10 \%$. 
CUADRO 4

El índice de Theil: estimador de sistema del MGM

\begin{tabular}{|c|c|c|c|c|c|c|c|}
\hline Variable & (1) & (2) & (3) & (4) & (5) & (6) & (7) \\
\hline Theil $_{i, t-1}$ & $\begin{array}{c}0,3181^{\mathrm{a}} \\
(0,1193) \\
{[2,6657]}\end{array}$ & $\begin{array}{c}0,2255^{\mathrm{c}} \\
(0,1356) \\
{[1,6626]}\end{array}$ & $\begin{array}{c}0,0226 \\
(0,2456) \\
{[0,0922]}\end{array}$ & $\begin{array}{c}0,3322^{\mathrm{c}} \\
(0,1735) \\
{[1,9143]}\end{array}$ & $\begin{array}{c}0,1798 \\
(0,1391) \\
{[1,2932]}\end{array}$ & $\begin{array}{c}0,2365^{\mathrm{c}} \\
(0,1378) \\
{[1,7151]}\end{array}$ & $\begin{array}{c}0,2186 \\
(0,2327) \\
{[0,9394]}\end{array}$ \\
\hline $\operatorname{OPEN}_{i, t-1}$ & $\begin{array}{c}-0,4547^{\mathrm{b}} \\
(0,2170) \\
{[-2,0950]}\end{array}$ & $\begin{array}{c}-0,7298^{\mathrm{b}} \\
(0,2897) \\
{[-2,5188]}\end{array}$ & $\begin{array}{c}-0,7865^{\mathrm{c}} \\
(0,4439) \\
{[-1,7717]}\end{array}$ & $\begin{array}{c}-0,4683^{\mathrm{c}} \\
(0,2640) \\
{[-1,7740]}\end{array}$ & $\begin{array}{c}-1,1196^{a} \\
(0,2870) \\
{[-3,9001]}\end{array}$ & $\begin{array}{c}-0,8191^{\mathrm{a}} \\
(0,2673) \\
{[-3,0640]}\end{array}$ & $\begin{array}{r}-0,6154^{\mathrm{c}} \\
(0,3487) \\
{[-1,7649]}\end{array}$ \\
\hline $\mathrm{PAT}_{i, t-1}$ & $\begin{array}{c}-0,3656^{\mathrm{c}} \\
(0,2069) \\
{[-1,7664]}\end{array}$ & $\begin{array}{c}-0,3671 \\
(0,2517) \\
{[-1,4584]}\end{array}$ & $\begin{array}{c}-0,1386 \\
(0,3377) \\
{[-0,4104]}\end{array}$ & $\begin{array}{c}-0,2108 \\
(0,1652) \\
{[-1,2763]}\end{array}$ & $\begin{array}{c}-0,4275 \\
(0,3680) \\
{[-1,1617]}\end{array}$ & $\begin{array}{c}-0,5312^{c} \\
(0,3005) \\
{[-1,7678]}\end{array}$ & $\begin{array}{c}-0,2733 \\
(0,2936) \\
{[-0,9308]}\end{array}$ \\
\hline $\mathrm{FD}_{i, t-1}$ & $\begin{array}{c}-1,4097^{b} \\
(0,6760) \\
{[-2,0853]}\end{array}$ & $\begin{array}{c}-1,5366^{\mathrm{b}} \\
(0,5957) \\
{[-2,5791]}\end{array}$ & $\begin{array}{c}-1,4353^{c} \\
(0,7599) \\
{[-1,8885]}\end{array}$ & $\begin{array}{c}-0,4021 \\
(0,6474) \\
{[-0,6212]}\end{array}$ & $\begin{array}{c}-1,9878^{a} \\
(0,7372) \\
{[-2,6963]}\end{array}$ & $\begin{array}{c}-1,4884^{\mathrm{c}} \\
(0,7821) \\
{[-1,9029]}\end{array}$ & $\begin{array}{r}-1,5142^{\mathrm{c}} \\
(0,7964) \\
{[-1,9012]}\end{array}$ \\
\hline $\mathrm{MW}_{i, t-1}$ & & $\begin{array}{c}-0,1909^{c} \\
(0,1065) \\
{[-1,7914]}\end{array}$ & & & & & \\
\hline $\mathrm{UNE}_{i, t-1}$ & & & $\begin{array}{c}3,4700^{c} \\
(1,9531) \\
{[1,7766]}\end{array}$ & & & & \\
\hline $\mathrm{BF}_{i, t-1}$ & & & & $\begin{array}{c}-0,4888^{c} \\
(0,2491) \\
{[-1,9624]}\end{array}$ & & & \\
\hline $\mathrm{PC}_{i, t-1}$ & & & & & $\begin{array}{c}0,0516^{\mathrm{b}} \\
(0,0254) \\
{[2,0319]}\end{array}$ & & \\
\hline $\operatorname{SOC}_{i, t-1}$ & & & & & & $\begin{array}{c}5,8652^{\mathrm{c}} \\
(3,2236) \\
{[1,8194]}\end{array}$ & \\
\hline $\mathrm{CORR}_{i, t-1}$ & & & & & & & $\begin{array}{c}0,0027^{\mathrm{c}} \\
(0,0015) \\
{[1,7249]}\end{array}$ \\
\hline Estadístico $J$ & $\begin{array}{l}30,3120 \\
p>0,14\end{array}$ & $\begin{array}{l}23,3662 \\
p>0,35\end{array}$ & $\begin{array}{l}14,6388 \\
p>0,45\end{array}$ & $\begin{array}{l}25,4334 \\
\mathrm{p}>0,14\end{array}$ & $\begin{array}{l}12,1678 \\
p>0,80\end{array}$ & $\begin{array}{l}14,0110 \\
\mathrm{p}>0,80\end{array}$ & $\begin{array}{c}9,0667 \\
p>0,75\end{array}$ \\
\hline $\mathrm{m}^{2}$ & $-0,1610$ & $-0,1606$ & 0,3007 & $-0,1500$ & $-0,1108$ & $-0,1755$ & $-0,1149$ \\
\hline Valor $p$ & 0,5785 & 0,6079 & 0,2603 & 0,6011 & 0,6855 & 0,4884 & 0,6647 \\
\hline Instrumentos & 27 & 27 & 20 & 24 & 23 & 25 & 18 \\
\hline
\end{tabular}

Fuente: elaboración propia.

Nota: los errores estándar están entre paréntesis y el valor $t$ está entre corchetes.

MGM: método generalizado de momentos.

a Significativo a un nivel del $1 \%$.

b Significativo a un nivel del $5 \%$.

c Significativo a un nivel del $10 \%$.

significativo y tiene signo positivo. Esto denota que un alto nivel de desempleo está vinculado a una gran desigualdad. El efecto negativo y significativo de esta variable no deja ninguna duda sobre la relación entre las variables.

La serie de variables que incorpora la influencia de los factores políticos en la desigualdad presenta resultados interesantes. Los coeficientes de PC contradicen la idea habitual de que un cambio más frecuente en el poder político reduce la desigualdad. Una posible explicación de este resultado reside en la perturbación de la continuidad de los programas sociales que podrían causar los cambios políticos sucesivos. Otra posible explicación consiste en el hecho de que, cuando un partido político logra aminorar la desigualdad de los ingresos, tiene una mayor probabilidad de permanecer en el poder. A primera vista, el signo positivo de soc puede parecer contrario a lo esperado. Sin embargo, el resultado se ajusta a la idea de que quizás estos recursos no lleguen a las familias más pobres (Feldstein, 1974; Browning y Browning, 1994; Mazza, 2001; Perry y otros, 2006). Finalmente, los coeficientes de CORR, que son positivos y estadísticamente significativos en los tres modelos, indican que hay una correlación entre el aumento de la corrupción y el agravamiento de la desigualdad de los ingresos. 
CUADRO 5

El índice 10/40: estimador de sistema del MGM

\begin{tabular}{|c|c|c|c|c|c|c|c|}
\hline Variable & (1) & (2) & (3) & (4) & (5) & (6) & (7) \\
\hline $10 / 40_{i, t-1}$ & $\begin{array}{l}0,3202^{\mathrm{c}} \\
(0,1651) \\
{[1,9388]}\end{array}$ & $\begin{array}{c}0,2761 \\
(0,1570) \\
{[1,7577]}\end{array}$ & $\begin{array}{c}0,0840 \\
(0,2000) \\
{[0,4203]}\end{array}$ & $\begin{array}{c}0,1089 \\
(0,1408) \\
{[0,7733]}\end{array}$ & $\begin{array}{c}0,1584 \\
(0,1581) \\
{[1,0015]}\end{array}$ & $\begin{array}{l}0,3354^{\mathrm{b}} \\
(0,1337) \\
{[2,5085]}\end{array}$ & $\begin{array}{c}0,4430^{\mathrm{b}} \\
(0,1869) \\
{[2,3695]}\end{array}$ \\
\hline OPEN $_{i, t-1}$ & $\begin{array}{c}-0,2878^{a} \\
(0,0856) \\
{[-3,3601]}\end{array}$ & $\begin{array}{c}-0,3318^{b} \\
(0,0881) \\
{[-3,7663]}\end{array}$ & $\begin{array}{c}-0,2465^{b} \\
(0,1201) \\
{[-2,0519]}\end{array}$ & $\begin{array}{c}-0,1607^{b} \\
(0,0636) \\
{[-2,5247]}\end{array}$ & $\begin{array}{c}-0,3259^{a} \\
(0,0932) \\
{[-3,4957]}\end{array}$ & $\begin{array}{c}-0,1482^{b} \\
(0,0659) \\
{[-2,2482]}\end{array}$ & $\begin{array}{c}-0,3721^{\mathrm{a}} \\
(0,0840) \\
{[-4,4301]}\end{array}$ \\
\hline $\operatorname{PAT}_{i, t-1}$ & $\begin{array}{c}-0,1512^{\mathrm{c}} \\
(0,0771) \\
{[-1,9609]}\end{array}$ & $\begin{array}{c}-0,1893^{b} \\
(0,1081) \\
{[-1,7511]}\end{array}$ & $\begin{array}{c}-0,1182 \\
(0,1070) \\
{[-1,1051]}\end{array}$ & $\begin{array}{c}-0,1827^{\mathrm{a}} \\
(0,0639) \\
{[-2,8562]}\end{array}$ & $\begin{array}{c}-0,2285^{\mathrm{c}} \\
(0,1332) \\
{[-1,7145]}\end{array}$ & $\begin{array}{c}-0,1670^{c} \\
(0,0865) \\
{[-1,9301]}\end{array}$ & $\begin{array}{c}-0,1368 \\
(0,1076) \\
{[-1,2707]}\end{array}$ \\
\hline $\mathrm{FD}_{i, t-1}$ & $\begin{array}{r}-0,3983^{c} \\
(0,2289) \\
{[-1,7399]}\end{array}$ & $\begin{array}{c}-0,7164^{b} \\
(0,3438) \\
{[-2,0838]}\end{array}$ & $\begin{array}{c}-0,6747^{\mathrm{b}} \\
(0,3047) \\
{[-2,2141]}\end{array}$ & $\begin{array}{c}-0,1495 \\
(0,1864) \\
{[-0,8020]}\end{array}$ & $\begin{array}{c}-0,6786^{b} \\
(0,2797) \\
{[-2,4257]}\end{array}$ & $\begin{array}{c}-0,4622^{c} \\
(0,2485) \\
{[-1,8600]}\end{array}$ & $\begin{array}{c}-0,1130 \\
(0,2864) \\
{[-0,3946]}\end{array}$ \\
\hline $\mathrm{MW}_{i, t-1}$ & & $\begin{array}{c}-0,0684^{c} \\
(0,0365) \\
{[-1,8727]}\end{array}$ & & & & & \\
\hline $\mathrm{UNE}_{i, t-1}$ & & & $\begin{array}{c}0,9448^{\mathrm{c}} \\
(0,5678) \\
{[1,6639]}\end{array}$ & & & & \\
\hline $\mathrm{BF}_{i, t-1}$ & & & & $\begin{array}{c}-0,2582^{a} \\
(0,0820) \\
{[-3,1482]}\end{array}$ & & & \\
\hline $\mathrm{PC}_{i, t-1}$ & & & & & $\begin{array}{l}0,0219^{\mathrm{b}} \\
(0,0105) \\
{[2,0787]}\end{array}$ & & \\
\hline $\operatorname{SOC}_{i, t-1}$ & & & & & & $\begin{array}{c}0,0568 \\
(0,7209) \\
{[0,0788]}\end{array}$ & \\
\hline $\operatorname{CORR}_{i, t-1}$ & & & & & & & $\begin{array}{c}0,0012^{\mathrm{a}} \\
(0,0004) \\
{[2,9981]}\end{array}$ \\
\hline Estadístico $J$ & $\begin{array}{l}21,5093 \\
p>0,25\end{array}$ & $\begin{array}{l}13,7063 \\
p>0,80\end{array}$ & $\begin{array}{l}11,1198 \\
p>0,85\end{array}$ & $\begin{array}{l}26,5805 \\
p>0,14\end{array}$ & $\begin{array}{l}14,5990 \\
p>0,65\end{array}$ & $\begin{array}{l}27,1170 \\
p>0,15\end{array}$ & $\begin{aligned} 8,7405 \\
\mathrm{p}>0,80\end{aligned}$ \\
\hline $\mathrm{m}^{2}$ & $-0,0359$ & $-0,0637$ & 0,1127 & $-0,0800$ & $-0,0119$ & $-0,0573$ & 0,0274 \\
\hline Valor $p$ & 0,8297 & 0,7042 & 0,6239 & 0,5891 & 0,9375 & 0,7123 & 0,8536 \\
\hline Instrumentos & 23 & 24 & 22 & 25 & 23 & 26 & 19 \\
\hline
\end{tabular}

Fuente: elaboración propia.

Nota: los errores estándar están entre paréntesis y el valor $t$ está entre corchetes. MGM: método generalizado de momentos.

a Significativo a un nivel del $1 \%$.

b Significativo a un nivel del $5 \%$.

c Significativo a un nivel del $10 \%$.

\section{IV}

\section{Conclusiones}

En este estudio se presentan datos empíricos relativos a la desigualdad de los ingresos en la economía del Brasil. El análisis regional muestra diferencias considerables entre las diversas regiones del país. En general, el sur presenta la menor desigualdad de los ingresos y los mejores indicadores. En cambio, el nordeste registró los peores datos a lo largo del período considerado. Sin embargo, la puesta en práctica de políticas sociales, como el programa Bolsa Família, se vincula a un progreso en todas las regiones. 
Los resultados del análisis empírico indican que hay varios factores que pueden explicar la reciente disminución de la desigualdad en el Brasil, como por ejemplo, la apertura comercial. Según señalan Rodrick (1997); Easterly (2005) y Nissanke y Thorbecke (2006), en las economías con un sector exportador intensivo en mano de obra, como la del Brasil, un incremento de la apertura comercial contribuye a que disminuya la desigualdad de los ingresos. Por lo tanto, la ampliación de los acuerdos comerciales y la optimización de la armonización fiscal con el objeto de fomentar la apertura comercial podrían constituir un mecanismo para hacer frente a la desigualdad. Los resultados también indican que el incremento del progreso tecnológico da lugar a una menor desigualdad de los ingresos, en consonancia con la tesis de Acemoglu (2002). También existe una correlación entre el desarrollo financiero y la reducción de la desigualdad (Liang, 2006; Ang, 2010). En consecuencia, resulta recomendable poner en práctica políticas que intensifiquen la colaboración entre empresas y universidades y que promuevan un entorno jurídico capaz de estimular la inversión en tecnología del sector privado.

La estrategia del Gobierno del Brasil de implementar aumentos reales del salario mínimo muestra una correlación negativa con la desigualdad; en cambio, el desempleo hace que esta se acreciente, de modo que es esencial elaborar políticas para eliminar el desempleo. Por otra parte, los resultados indican que el programa Bolsa Família se relaciona con una retracción de la desigualdad de los ingresos.

Los datos empíricos acerca del efecto de los factores políticos en la desigualdad indican que un cambio en el poder político no contribuye a que mejore la situación social. De forma similar, el incremento del gasto social no hace que aminore la desigualdad de los ingresos. Una posible explicación reside en el hecho de que estos recursos no llegan a las familias más pobres (Feldstein, 1974; Browning y Browning, 1994; Mazza, 2001; Perry y otros, 2006). El uso de herramientas específicas contra la pobreza podría lograr mejores resultados. Finalmente, el caso del Brasil confirma la suposición de que una mayor corrupción se relaciona con el aumento de la desigualdad (Gupta, Davoodi y Alonso-Terme, 2002).

En resumen, los datos empíricos de este estudio permiten observar que es posible mejorar la lucha contra la desigualdad mediante la combinación de una mayor apertura comercial, el desarrollo tecnológico y financiero, la reducción de la tasa de desempleo, la implementación de políticas sociales con efecto directo en las familias más pobres y la utilización de mecanismos que restrinjan la corrupción.

\section{Bibliografía}

Acemoglu, D. (2002), "Technical change, inequality, and the labor market", Journal of Economic Literature, vol. 40, $\mathrm{N}^{\circ} 1$, Nashville, Tennessee, American Economic Association.

Acemoglu, D. y A. Newman (2002), "The labour market and corporate structure", European Economic Review, vol. 46, $\mathrm{N}^{\circ} 10$, Amsterdam, Elsevier.

Adams, S. (2008), "Globalization and income inequality: implications for intellectual property rights", Journal of Policy Modeling, vol. 30, $\mathrm{N}^{\circ}$ 5, Amsterdam, Elsevier.

Adelman, I. y C. Morris (1965), "A factor analysis of the interrelationship between social and political variables and per capita gross national product", Quarterly Journal of Economics, vol. 79, $\mathrm{N}^{\circ}$ 4, Oxford, Oxford University Press.

Aghion, P. y P. Bolton (1997), "A theory of trickle-down growth and development", Review of Economic Studies, vol. 64, N ${ }^{\circ} 2$, Wiley Blackwell.

Alonso-Borrego, C. y M. Arellano (1998), "Symmetrically normalized instrumental-variable estimation using panel data", CEMFI Working Paper, $\mathrm{N}^{\circ} 9612$, septiembre.

Alt, J. y D. Lassen (2010), "Enforcement and public corruption: evidence from US states", EPRU Working Paper Series, $\mathrm{N}^{\circ}$ 2010-08, Copenhague, Universidad de Copenhague.

Ang, J.B. (2010), "Finance and inequality: the case of India", Southern Economic Journal, vol. 76, $\mathrm{N}^{\circ}$ 3, Southern Economic Association.

Apergis, N., O.C. Dincer y J. Payne (2010), “The relationship between corruption and income inequality in U.S. states: evidence from a panel cointegration and error correction model", Public Choice, vol. 145, $\mathrm{N}^{\circ} 1$, Springer.
Arellano, M. (2003), Panel Data Econometrics, Oxford, Oxford University Press.

Arellano, M. y S. Bond (1991), "Some tests of specification for panel data: Monte Carlo evidence and an application to employment equations", Review of Economic Studies, vol. 58, № 2, Oxford, Oxford University Press.

Arellano, M. y O. Bover (1995), "Another look at the instrumental variable estimation of error-components model", Journal of Econometrics, vol. 68, $\mathrm{N}^{\circ}$ 1, Amsterdam, Elsevier.

Blinder, A.S. y H.Y. Esaki (1978), "Macroeconomic activity and income distribution in the postwar United States", Review of Economics and Statistics, vol. 60, $\mathrm{N}^{\circ} 4$, Cambridge, Massachusetts, The MIT Press.

Blundell, R. y S. Bond (1998), "Initial conditions and moment restrictions in dynamic panel data models", Journal of Econometrics, vol. 87, $\mathrm{N}^{\circ} 1$, Amsterdam, Elsevier.

Boll, J.L.S. (2010), "A corrupção governamental no Brasil: construção de indicadores e análise da sua incidência relativa nos estados brasileiros", Brasilia [en línea] http://www.cgu. gov.br/concursos/Arquivos/5_ConcursoMonografias/MHprofissionais-jose-luis-serafini-boll.pdf.

Bond, S.R., A. Hoeffler y J. Temple (2001), "GMM estimation of empirical growth models", CEPR Discussion Paper, $\mathrm{N}^{\circ} 3048$, Londres, Centre for Economic Policy Research.

Browning, E. y J.M. Browning (1994), Public Finance and the Price System, Englewood Cliffs, Prentice Hall.

Claessens, S. y E. Perotti (2005), "The links between finance and inequality: channels and evidence", World Development Report Background Papers, Washington, D.C., Banco Mundial. 
Dincer, O.C. y B. Gunalp (2008), "Corruption, income inequality and poverty in the United States", Nota di lavoro, $\mathrm{N}^{\circ}$ 54, Fondazione Eni Enrico Mattei.

Easterly, W. (2005), "National policies and economic growth: a reappraisal", Handbook of Economic Growth, Philippe Aghion y Steven Durlauf (eds.), Amsterdam, Elsevier.

Engel, E., A. Galetovic y C. Raddatz (1999), "Taxes and income distribution in Chile: some unpleasant redistributive arithmetic", Journal of Development Economics, vol. 59, $\mathrm{N}^{\circ} 1$, Amsterdam, Elsevier.

Fang, C., L. Huang y M. Wang (2008), "Technology spillover and wage inequality", Economic Modelling, vol. 25, $\mathrm{N}^{\circ} 1$, Amsterdam, Elsevier.

Feldstein, M. (1974), "Social security, induced retirement, and aggregate capital accumulation", Journal of Political Economy, vol. 82, $\mathrm{N}^{\circ}$ 5, Chicago, The University of Chicago Press.

Forteza, A. e I. Rossi (2009), "The contribution of government transfer programs to inequality: A net-benefit approach", Journal of Applied Economics, vol. 12, $\mathrm{N}^{\circ}$ 1, Amsterdam, Elsevier.

Galor, O. y J. Zeira (1993), "Income distribution and macroeconomics", Review of Economic Studies, vol. 60, N ${ }^{\circ}$, Blackwell Wiley.

Gasparini, L. (2003), "Different lives: inequality in Latin America the Caribbean", Inequality the State in Latin America and the Caribbean, Washington, D.C., Banco Mundial.

Goñi, E., J. López y L. Servén (2011), "Fiscal redistribution and income inequality in Latin America", World Development, vol. 39, $\mathrm{N}^{\circ}$ 9, Amsterdam, Elsevier.

Greenwood, J. y B. Jovanovic (1990), "Financial development, growth and, the distribution of income", Journal of Political Economy, vol. 98, $\mathrm{N}^{\circ} 5$, Chicago, The University of Chicago Press.

Gupta, S., H. Davoodi y R. Alonso-Terme (2002), "Does corruption affect income inequality and poverty?", Economics of Governance, vol. 3, $\mathrm{N}^{\circ} 1$, Springer.

Kumar, A. (2005), "Access to financial services in Brazil", Directions in Development, Washington, D.C., Banco Mundial.

Lemos, S. (2009), "Minimum wage effects in a developing country", Labour Economics, vol. 16, $\mathrm{N}^{\circ} 2$, Amsterdam, Elsevier.
Levitt, S. y J. Poterba (1999), "Congressional distributive politics and state economic performance", Public Choice, vol. 99, $\mathrm{N}^{\circ} 1-2$, Springer.

Liang, Z. (2006), "Financial development and income distribution: a system GMM panel analysis with application to urban China", Journal of Economic Development, vol. 31, $\mathrm{N}^{\circ} 2$.

Madsen, J. (2007), "Technology spillover through trade and TFP convergence: 135 years of evidence for the OECD countries", Journal of International Economics, vol. 72, $\mathrm{N}^{\circ} 2$, Amsterdam, Elsevier.

Martínez, R., L. Ayala y J. Ruiz-Huerta (2001), "The impact of unemployment on inequality and poverty in OECD countries", Economics of Transition, vol. 9, $\mathrm{N}^{\circ}$ 2, Londres, Banco Europeo de Reconstrucción y Desarrollo.

Mazza, J. (2001), "Unemployment insurance: case studies and lessons for Latin America and the Caribbean", Documento de Trabajo, $\mathrm{N}^{\circ}$ 411, Washington, D.C., Departamento de Investigación, Banco Interamericano de Desarrollo.

Meschi, E. y M. Vivarelli (2009), "Trade and income inequality in developing countries", World Development, vol. $37, \mathrm{~N}^{\circ} 2$, Amsterdam, Elsevier.

Nissanke, M. y H. Thorbecke (2006), "Channels and policy debate in the globalization-inequality-poverty nexus", World Development, vol. 34, $\mathrm{N}^{\circ} 8$, Amsterdam, Elsevier.

Perry, G. y otros (2006), Poverty Reduction and Growth: Virtuous and Vicious Circles, Washington, D.C., Banco Mundial.

Rodrik, D. (1997), Has Globalization Gone Too Far?, Washington, D.C., Institute for International Economics.

Roine, J., J. Vlachos y D. Waldenström (2009), "The long-run determinants of inequality: what can we learn from top income data?", Journal of Public Economics, vol. 93, № 7-8, Amsterdam, Elsevier.

Rupasingha, A. y S. Goetz (2007), "Social and political forces as determinants of poverty: a spatial analysis", Journal of SocioEconomics, vol. 36, $\mathrm{N}^{\circ} 4$, Amsterdam, Elsevier.

Staiger, D. y J.H. Stock (1997), "Instrumental variables regression with weak instruments", Econometrica, vol. 65, ํㅜ 3, Nueva York, The Econometric Society.

Wooldridge, J. (2001), Econometric Analysis of Cross Section and Panel Data, Cambridge, Massachusetts, The MIT Press. 